\title{
Using LSB Method For Hiding Hill Encrypted Grayscale And RGB Images In RGB Image
}

\author{
${ }^{1}$ Huda adel ali , ${ }^{2}$ Enas wahab Abood,${ }^{3}$ Wafaa A. Khudhair \\ ${ }^{1}$ Collage of computer science and information technology,/university of Basrah \\ ${ }^{2}$ Collage of Science , university of Basrah \\ ${ }^{3}$ Collage of Science , university of Basrah
}

\begin{abstract}
Availability of data anywhere and ease of access and interception the information in different forms as text ,images ,video...etc., create an important issue represented by data security both steganography and encryption was appeared to achieve this mission. In this paper a hybrid technique was used to secure messages of grayscale and colored images in RGB cover image by encrypting the message with Hill encrypting algorithm and hiding it by LSB method in RGB image with random locations generated by seed number and taking three LSB bits, many different sizes of images was experimented and results was analyzed by PSNR , Correlation and histogram analysis methods, all proves the efficiency of proposed technique in securing images .
\end{abstract}

\section{Introduction}

Steganography is used to hide message of data in other cover data using key ,so the cover data seemed irrelevant, the message and cover could be any type of data such as text, image, audio and video[1].only the sender and receiver who has the key is able to extract the message out of cover file. Steganography sometimes is applied for securing messages and for avoiding unwanted attention or uses in places where the usage of encryption is illegal[2]. Various methods have been proposed for image steg anography depending on the image data domain. The image domain is classified into two categories: - (a) Spatial Domain, and (b) Transform Domain. For spatial domain, the most widespread method and the simplest one is the LSB( Least_Significant_Bit), which substitutes the least significant bit of cover image pixels that selected to hide the message bits [3]. Transform or frequency domain techniques are depended on the manipulation of the orthogonal transform of the image rather than the image itself [4] including transforms like discrete cosine transform DCT, discrete Fourier transform DFT, Hartley Transform, etc.

The encryption from other hand is a way of transforming the overall view of understood data to disfigured one, so the encrypted messages seemed to be obvious holding important data needed to be figured out and it fails if the intruder able to decrypt the cipher message, At other hand Steganography fails to secure data when the attacker detects the presence of a secret data inside another cover file [5]. Thus, for more ensuring data security, steganography and Encryption is used together so any observer finds difficulty in realizing the presence of massages and if its retrieved it stills ununderstood and meaningless. In this paper, A hybrid approach of cryptography and steganography is proposed to strengthen security insurance for grayscale and colored images. The cryptography was implemented by using Hill cipher algorith $\mathrm{m}$ which is a polygraphic cipher algorith $\mathrm{m}$ based on linear transformation while a LSB was employed for steganography with a modification as a random spreading of bits and more than one least bit.

\section{Related Work:}

The data security system witnesses a continuous increasing in threats lately which makes the data security issue is a matter of concerning for the researchers and security experts. Many techniques of 
steganography and Cryptography are presented to defeat this threats. Many researchers nowadays blended both of these techniques for acquiring higher level of security[5]. Ankit Dhamija et.al. [5] proposed a strategy for cloud architecture called (Secure Cloud Migration Architecture using Cryptography and Steganography) SCMACS to secure information transmission from the organization of client to servers of the provider of Cloud Service (CSP) by combining approach of steganography and cryptography to provide two levels of security for transmitted data the data transformed to code with encryption algorith $\mathrm{m}$ then converted into image by using steganography.

Al-Mashadi H. M. and Khalaf A. A. [6] presented three efficient hybrid homomorphic encryption techniques to encrypt image for exchanging images in public cloud safely. The suggested methods are restricted to El-Gamal and EHC (Enhanced Ho mo morphic Cryptosystem) . the proposed system proves to be immunity and effective in security and time compared with El-Gamal and EHC schemes. Abduljaleel I. Q.[7] proposed a system co mbined of two techniques for securing color images by encrypting the secret image using Integer Wavelet Transform (IWT) and scrambled it with Arnold transform then xored it with key of chaotic map, For more security the secret encrypted image has been hidden in a color image as a cover by using (LSB) method. Sevierda R. et.al.[2] describes the effectiveness of Steganography for secret messages of grayscale images hidden in a cover RGB_images, the secret image is encrypted by using rubik's cube principle based method that moves the pixels position of digital image. To obtain more security level, LSB method used for hiding encrypted image. Efficiency of the suggested scheme is verified using analysis schemes like histogram analysis, Brute-Force attack, Avalanche effect, visual attack and analysis of Chi-Square statistical attack .

Astuti Y. P. et.al.[8] Proposed a safe and simple method for hid ing messages using LSB techniques with a modification to avoid the predictability of LSB. XOR operation is implemented three times to encrypt the message then embedded on the LSB. To simplify the encryption and decryption processes of message,the keys in XOR operations are used by three MSB bits. It provides security for messages with a simple operation ,the softness quality of the stego image scores a PSNR value above $50 \mathrm{~dB}$. Gotfried C. Prasetya di et.al.[1]they produced steganography method called append insertion which hides any type of computer file in a carrier or cover certain type computer file for removing the restrictions on message file type .AES_256 algorith $m$ is used for encryption with passphrase. For identification and verification of orig inal message a block of bytes is specialized. This system was tested using five secret files of different types as a messages. A SHS_256 is used to calculate files integrity before being hidden and after retrieving that produce exact hash values. Bairagi [9] produced a security system using even-odd encryption method depends on ASCII code and converting the encrypted massage with Gray code then embedding the message in a picture to burden cryptanalyst's job. Qiang Zhang et al. [10] proposed algorithm based on DNA sequence addition combining with chaos method for image encryption .The secret image is encoded by DNA sequence matrix, then this matrix segmented and added togather, A complement operation is accomplished using two Logistic maps to the DNA sequence and DNA sequence matrix that produced is decoded to obtain the encrypted image.

\section{Proposed System}

A hybrid technique in this paper is contained two phases:

1. Encryption phase: the system accepts a secret message of grayscale or RGB image to be encrypted using Hill Algorithm that based on a linear transformation with Key of $n \times n$ integer numbers matrix , the key is a non-singular matrix which $\mathrm{n}$ is the size of the block that the message is divided to Hill Cipher algorithm can resist the frequency analysis, high speed and high throughput [11].

The message or data of image called $\mathrm{I}$ is encrypted as:

C_I $=$ KI mod 256

Where 256 is the largest value of image pixel, C_I is the cipher image block .

The decryption of cipher image $C_{-} I$ to produce secret image $I$ is obtained by using inverse matrix of the encryption key $\mathrm{K}$,

I $=K^{-1}$ C_I $\bmod 256$ 
2. Steganography phase : to secure an encrypted grayscale image, the 8-bits of each pixel in secret encrypted image is spread in one pixel of selected RGB cover image that contain 24 bits of three level of colors, as in fig(1):

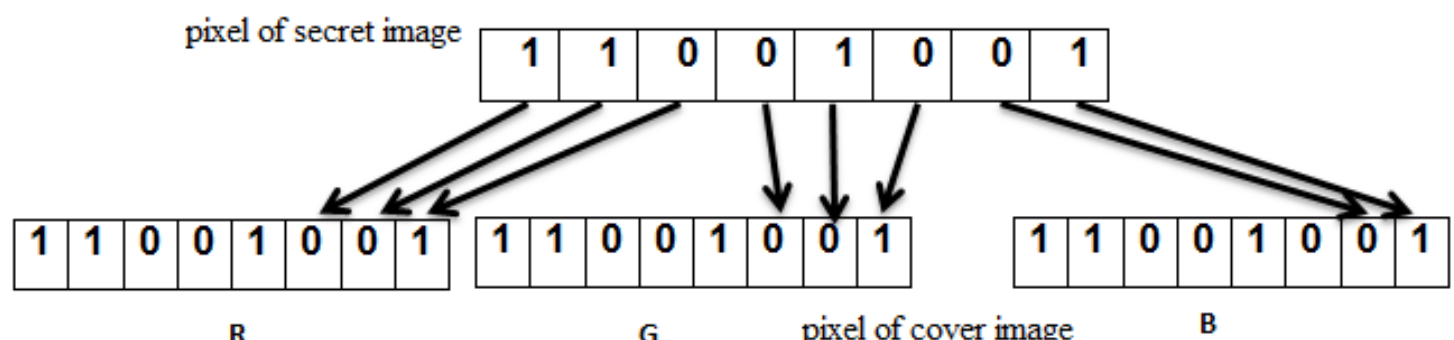

fig(1) explaining the way of hiding secret 8-bit pixel in 24-bit pixel of cover

The cover image is separated into three main color matrix R,G,B , for each pixel of secret image the three LSB of R cover is replaced with three MSB of secret pixel, Three LSB of G cover holds the three second bits as in fig(1) and the two LSB of secret pixel embedded in two LSB of B cover.

For secret RGB image a simple process is done to transform it into 2D matrix of 8-bits pixel before being encrypted and hidden that separating $\mathrm{R}, \mathrm{G}$ and $\mathrm{B}$ colors then reconstruct a new image by adding a new rows at the end of $R$ matrix represents $G$ matrix then add $B$ matrix as in fig(2):

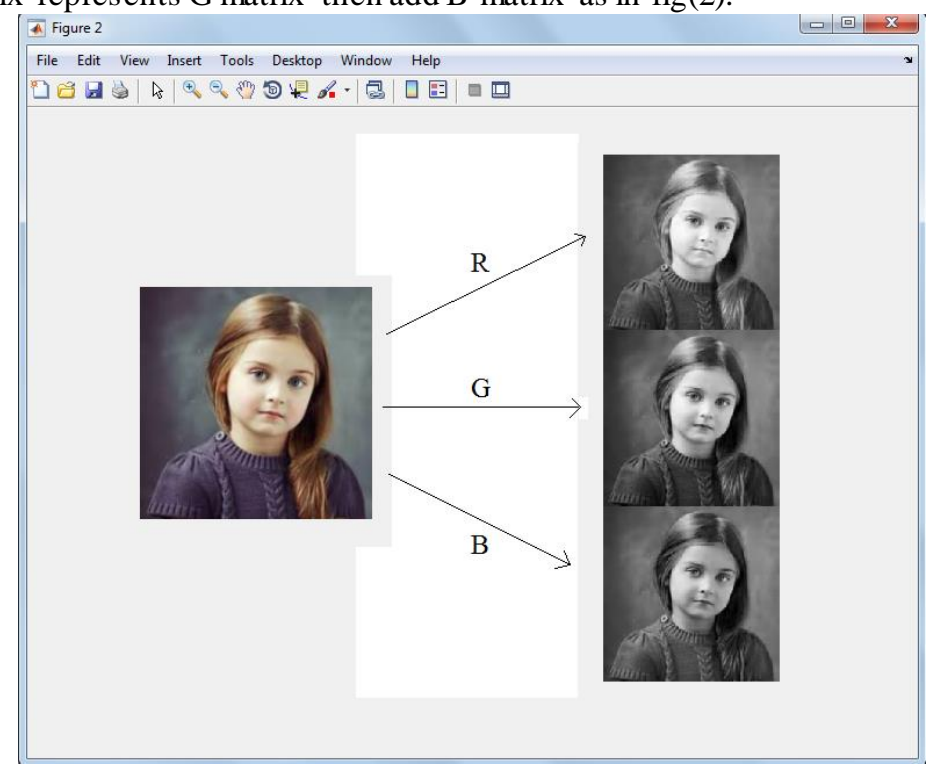

Fig(2) transform RGB image to 2D image

The way of choosing pixels from cover image for hide secret pixels is done randomly through using summation of encryption key matrix $\mathrm{K}$ as a seed to generate a series of locations rows and columns to hide secret message pixels in cover image then the encryption key $\mathrm{K}$ and secret image information like its dimensions and type (Gray or RGB) image is transformed to text the text is separated by symbol \% then use another Key K_H which is an integer number to hide encryption key and secret image information in cover image as in fig (3). 


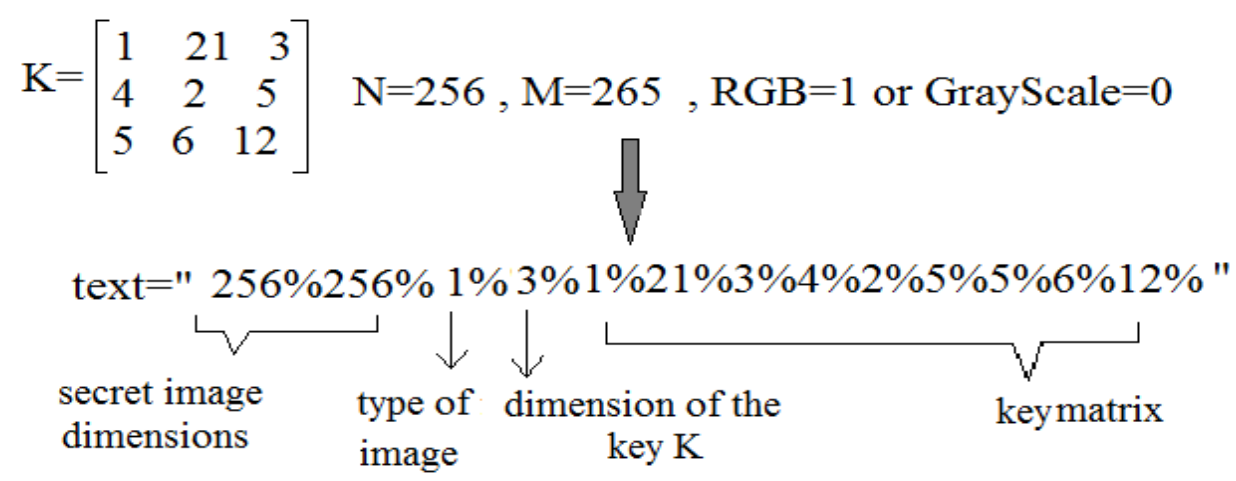

Fig(3) transformation the key $\mathrm{K}$ and image information into text to be hidden in cover file

In the receiver side the key $\mathrm{K} \_\mathrm{H}$ is used for generation the random location of the encryption key $\mathrm{K}$ and secret image information then the summation of the key $\mathrm{K}$ used to unhide the secret pixels as a matrix.

4. Experimental Results Analysis:

Many different size and types of (gray or RGB) secret images was encrypted and hidden in various RGB images with proposed system and three types of security analysis methods was used for testing system effectiveness

1. Histogram analysis:

Histogram is used for showing the frequency allocation of pixel weights in an image, in this paper histogram was used for ciphered image to show its uniformed that make it resistible for statistical attack, fig(4).
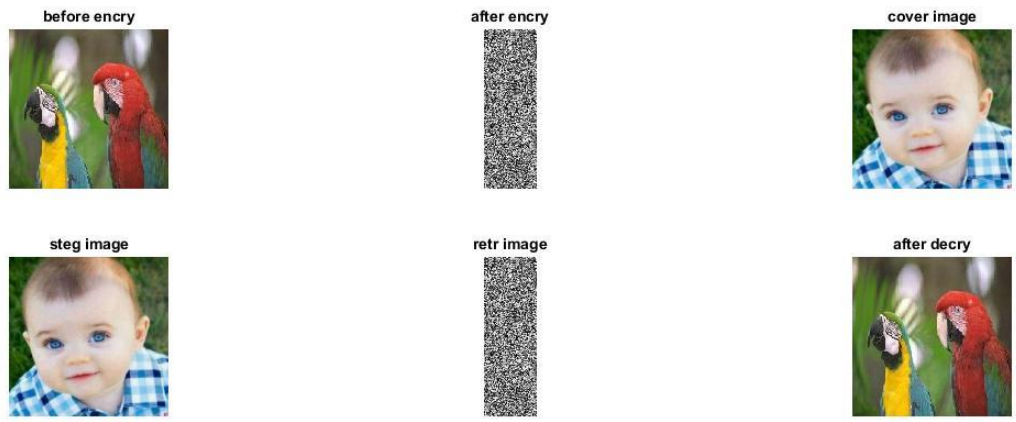

PSNR for cover Vs. hidding $=52.8836$

PSNR before and after encrypt $=8.6027$

PSNR for image Vs. encryption= Inf
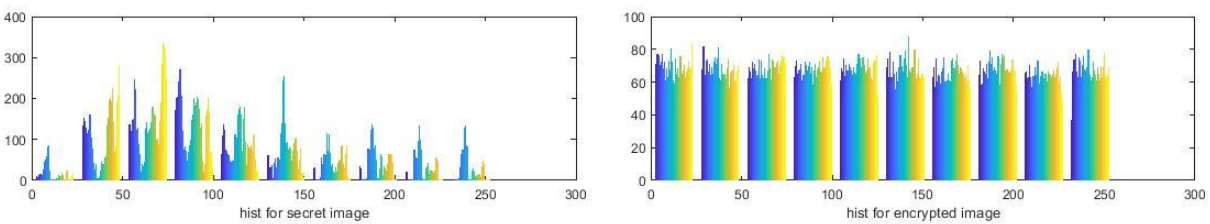

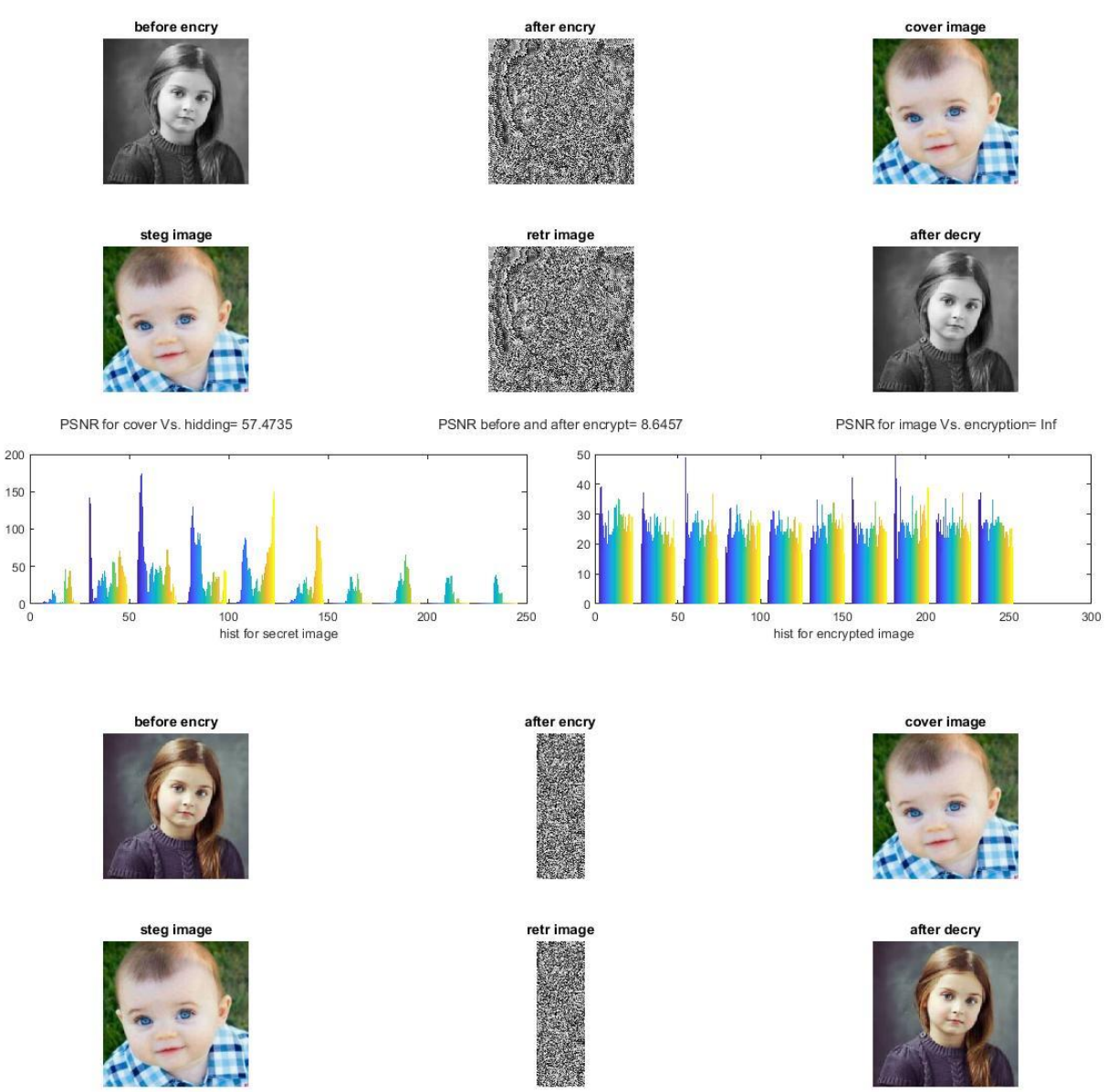

PSNR for cover Vs. hidding $=52.6685$

PSNR before and after encrypt $=8.7854$
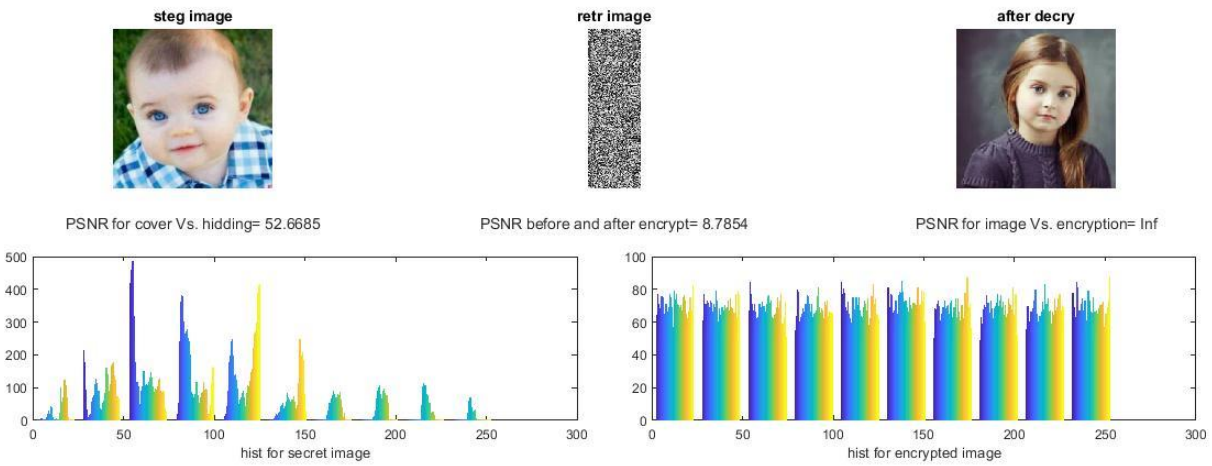

Fig(4) the histogram for secret plain image Vs. encrypted image

2. PSNR Analysis:

PSNR is most common analysis based on mean squared error (MSE) between two images such as plant and ciphered or cover images before and after hiding to show the noise approximation scale .MSE and PSNR(in $\mathrm{dB}$ ) is calculated as :

$$
\begin{gathered}
M S E=\frac{1}{M N} \sum_{k=1}^{M} \sum_{h=1}^{N}(I(k, h)-I(k, h))^{2} \\
P S N R=10 * \log _{10} \frac{255}{\sqrt{M S E}}
\end{gathered}
$$

Thus, $I$ is a noise-free MN monochrome image and its noisy approximation $I$

The value of PSNR higher than $50 \mathrm{~dB}$ for 8 bits image is better, For 16-bit data most acceptable values for the PSNR are between 60 and $80 \mathrm{~dB}$. when the noise is absence, the two images $I$ and $I$ are matching, that leads MSE to be zero. In this case the PSNR value is undefined or infinite [12]. 
In this paper the PSNR was calculated for plain and encrypted images that was very low which means they differ a lot. The PSNR for secret image and reconstructed image was infinity leads to that the MSE is 0 and they are identical. Table(1).

Table(1) PSNR results between the original image and reconstructed image(grayscale and RGB)

\begin{tabular}{|l|l|l|l|l|}
\hline \multirow{2}{*}{ Image } & \multicolumn{3}{|l|}{ PSNR for plain grayscale image } & \multicolumn{2}{l|}{ PSNR for plain RGB image } \\
\cline { 2 - 5 } & $\begin{array}{l}\text { With encrypted } \\
\text { image }\end{array}$ & $\begin{array}{l}\text { With } \\
\text { reconstructed } \\
\text { image }\end{array}$ & $\begin{array}{l}\text { With encrypted } \\
\text { image }\end{array}$ & $\begin{array}{l}\text { With } \\
\text { reconstructed } \\
\text { image }\end{array}$ \\
\hline & 8.235 & Inf & 8.6027 & Inf \\
\hline & 9.308 & Inf & 9.55 & Inf \\
\hline
\end{tabular}

\section{Correlation Analysis}

Correlation analysis is widely used to measure the similarty between two images[13][ 14]., Here we use it between original and the cipher image. The correlation is

defined as follow :

$$
c=\frac{\sum_{h=1}^{N} \sum_{k=1}^{M}\left(I_{1}(h, k)-\overline{I_{1}}\right)\left(I_{2}(h, k)-\overline{I_{2}}\right)}{\sqrt{\sum_{h=1}^{N} \sum_{k=1}^{M}\left(I_{1}(h, k)-\overline{I_{1}}\right)^{2} * \sum_{h=1}^{N} \sum_{k=1}^{M}\left(I_{2}(h, k)-\overline{I_{2}}\right)^{2}}}
$$

Here $I_{1}(h, k)$ is pixel intensity of gray-scale original image, $I_{2}(h, k)$ is the gray-scale value of the cipher image, $\bar{I}_{1}$ and $\bar{I}_{2}$ are the mean values of the images $I_{1}$ and $I_{2}$ respectively.

For RGB Image we calculate the correlation between each color component then take their mean. The value of correlation ranged in [0,1], for identical images should be high close to 1 , whereas it decreases close to 0 as much as they differ, table(2) shows the results of correlation analysis between encrypted and plain images. 
Table(1) correlation coefficients between the original image and reconstructed image(grayscale and RGB)

\begin{tabular}{|l|l|l|l|l|}
\hline \multirow{2}{*}{ Image } & \multicolumn{2}{|l|}{ Correlation for plain grayscale image } & Correlation for plain RGB image \\
\cline { 2 - 5 } & $\begin{array}{l}\text { With encrypted } \\
\text { image }\end{array}$ & $\begin{array}{l}\text { With } \\
\text { reconstructed } \\
\text { image }\end{array}$ & $\begin{array}{l}\text { With encrypted } \\
\text { image }\end{array}$ & $\begin{array}{l}\text { With } \\
\text { reconstructed } \\
\text { image }\end{array}$ \\
\hline & 0.0137 & 1 & 0.0219 & 1 \\
\hline & 0.0127 & 1 & 0.0025 & 1 \\
\hline
\end{tabular}

\section{Conclusions}

The security of transferred data became an important issue because the ease of data access via transmitted channels, The security experts and researchers tend to use and develop many security aspects like cryptography and steganography. The algorith $\mathrm{m}$ proposed in this paper was combined of two methods encryption with Hill method and hiding by LSB technique for securing grays cale and RGB images in a cover file of RGB image. The analytical methods proved the efficiency of the system via testing many types and sizes of secret and cover image with condition the cover is always bigger than the secret. The time consumption for overall process (encryption and hiding for grayscale) was low, it ranged from $(0.0011)$ to $(0.0021)$ seconds for encryption and from (0.017) to (0.169) hid ing, while for (encryption and hiding for color image) was ranged from $(0.0141)$ to $(0.02)$ for encryption and from $(0.16)$ to $(0.2)$ hiding .Also the statistical results was very acceptable that for PSNR less than (10) d B that show the effectiveness for encryption technique and was (Infinity) dB for retrieved secret image which means it identical with no information loss.

The correlation analysis scores a degrees close to 0 for encrypted image Vs. original image means they not related to each other and immune against attacker analysis. At the other hand, the histogram analysis for ciphered images reflects its homogeneity that makes it opponent. From all analytical statistics results the systemproves to be an effective tool for securing images against attackers.

\section{References:}

1. Gotfried C. Prasetyadi ; Achmad Benny Mutiara ; Rina Refianti (2018),File encryption and hiding application based on advanced encryption standard (AES) and append insertion, steganography method . 2017 Second International Conference on Informatics and Computing (ICIC), Jayapura, Indonesia. 
2. Sevierda Raniprima ; Bambang Hidayat ; Nur Andini (2017).Digital image steganography with encryption based on rubik's cube principle. 2016 International Conference on Control, Electronics, Renewable Energy and Communications (ICCEREC). Bandung, Indonesia.

3. Praneeta D., \& Padma B.( 2014), Hiding Image in Image by using FMM with LSB Substitution in Image Steganography, International Journal of Advance Research in Computer Science and Management Studies, Vol. 2, Issue 11.

4. Snehal O.Mundhada, V. K.Shandilya (2012).Spatial and Transformation Domain Techniques for Image Enhancement. International Journal of Engineering Science and Innovative Technology (IJESIT) Volume 1, Issue 2, ISSN: 2319-5967.

5. Ankit Dhamija; Vijay Dhaka (2016).A Novel Cryptographic and Steganographic Approach for Secure Cloud Data Migration. 2015 International Conference on Green Computing and Internet of Things (ICGCIoT). 978-1-4673-7910-6/15/\$31.00, 2015 IEEE.

6. Haider M. Al-Mashadi, Ala'a A. Khalaf .(2018). Hybrid Homomorphic Cryptosystem For Secure Transfer Of Color Image On Public Cloud. Journal of Theoretical and Applied Information Technology. Vol.96. No 19. ISSN: 1992-8645.

7. Iman Qays Abduljaleel.(2016).Using IWT and LSB Method to Hide Encrypted image in Color image . Journal of Basrah Researches ((Sciences)) Vol. (42). No. (1) A.

8. Yani Parti Astuti ; De Rosal Ignatius Moses Setiadi ; Eko Hari Rachmawanto ; Christy Atika Sari (2018). Simple and secure image steganography using LSB and triple XOR operation on MSB. 2018 International Conference on Information and Communications Technology (ICOIACT). Publisher: IEEE. Yogyakarta, Indonesia.

9. Bairai, A. K.( 2011), ASCII based Even-Odd Cryptography with Gray code and Image Steganography: A dimension in Data Security, ISSN:2078-5828 (Print), ISSN 2218- 5224 (Online), Vol.01,No.2, , pp:37-41, Manuscript Code: 110112.

10. Qiang Zhang, Ling Guo, Xiaopeng Wei, (2010). Image encryption using DNA addition combining with chaotic maps. Mathematical and Computer Modelling, Vol. 52, PP. 2028-2035.

11. Adinarayana Reddy Ka, Vishnuvardhan Bb, Madhuviswanathame, Krishna A. V. N.d (2012) .A Modified Hill Cipher Based on Circulant Matrices. Procedia Technology 4(2012) 114 - 118. 22120173 () 2012 Published by Elsevier Ltd. doi: 10.1016/j.protcy.2012.05.016 .

12. Raouf Hamzaoui, Dietmar Saupe . (2006). Barni, Mauro, ed. Fractal Image Compression. Document and Image Compression. 968. CRC Press. pp. 168-169. ISBN 9780849335563. Retrieved 5 April 2011.

13. Haider M. Al-Mashhadi.(2017).Quality Assessment for Image Encryption Techniques using Fuzzy Logic System. International Journal of Computer Applications, Vol. 157, No 5 .

14. Haider M. Al-Mashhadi; Iman Q. Abduljaleel.(2017) .Color Image Encryption using Chaotic Maps, Triangular Scrambling, with DNA Sequences. 2017 International Conference on Current Research in Computer Science and Information Technology (ICCIT), Slemani - Iraq. 978-1-5386-2955-0/17/\$31.00 (C)2017 IEEE. 